\title{
Lexicographic allocations and extreme core payoffs: the case of assignment games
}

\author{
Marina Núñez • Tamás Solymosi
}

Received: date / Accepted: date

\begin{abstract}
We consider various lexicographic allocation procedures for coalitional games with transferable utility where the payoffs are computed in an externally given order of the players. The common feature of the methods is that if the allocation is in the core, it is an extreme point of the core. We first investigate the general relationships between these allocations and obtain two hierarchies on the class of balanced games.

Secondly, we focus on assignment games and sharpen some of these general relationships. Our main result shows that, similarly to the core and the coalitionally rational payoff set, also the dual coalitionally rational payoff set of an assignment game is determined by the individual and mixed-pair coalitions, and present an efficient and elementary way to compute these basic dual coalitional values. As a byproduct we obtain the coincidence of the sets of lemarals (vectors of lexicographic maxima over the set of dual coalitionally rational payoff vectors), lemacols (vectors of lexicographic maxima over the core) and extreme core points. This provides a way to compute the AL-value (the average of all lemacols) with no need to obtain the whole coalitional function of the dual assignment game.
\end{abstract}

Keywords Assignment game · Extremal core allocations · Average lexicographic value

The first author's research has been supported by grant ECO2014-52340-P of the Spanish Ministry of Science and Innovation and 2014SGR40 of the Government of Catalonia, and also partly supported by COST Action IC1205 on Computational Social Choice. The second author's research has been supported by OTKA grant K-101224, by the Hungarian Academy of Sciences under its Momentum Programme (LD-004/2010), and also partly supported by COST Action IC1205 on Computational Social Choice.

\section{Núñez}

Department of Mathematical Economics, Finance and Actuarial Sciences, University of Barcelona, Av. Diagonal, 690, 08034 Barcelona, Spain

Tel.: +34-93-4021991 Fax: +34-93-4034892 E-mail: mnunez@ub.edu

T. Solymosi

Department of Operations Research and Actuarial Sciences, Corvinus University of Budapest, and MTA-BCE 'Lendület' Strategic Interactions Research Group; Fővám tér 8, 1093 Budapest, Hungary

Tel.: +36-1-4827457 Fax: +36-1-4827430 E-mail: tamas.solymosi@uni-corvinus.hu 
Mathematics Subject Classification (2000) 91A12 $91 \mathrm{~B} 68$

\section{Introduction}

Assignment games (Shapley and Shubik, 1972) are models of assignment (twosided matching) markets with transferable utilities where the aim of each player on one side is to form a profitable coalition with a player on the other side. Since only such bilateral cooperations have worth, these games are completely defined by the matrix containing the cooperative worths of all possible pairings of players from the two sides.

Shapley and Shubik (1972) showed that the core of an assignment game is precisely the set of dual optimal solutions to the assignment optimization problem on the underlying matrix of mixed-pair profits. This result not only implies that all assignment games have a non-empty core but also that the core can be determined without explicitly generating the entire coalitional function of the game. Typically, the core of the assignment game contains infinitely many allocations which makes necessary some core selection.

The Shapley value (Shapley, 1953) is a well-known single-valued solution for coalitional games with transferable utility. This value is the average of the marginal payoff vectors, where the marginal payoff vector associated with a given ordering of the set of agents is defined by paying to each agent his marginal contribution to the set of predecessors in the ordering. In an assignment game, each extreme core allocation is a marginal payoff vector (Hamers et al., 2002). However, the converse is not true and this is why the Shapley value of an assignment game may not select a core allocation.

Another single-valued solution for coalitional games (with a non-empty core) is the AL-value (Tijs et al., 2011), that is defined as the average of the lexicographical maximum core allocations or lemacols ${ }^{1}$. Given an order of the agents, the corresponding lemacol is recursively defined by paying to each agent the maximum he can obtain inside the core under the restriction that his predecessors in the order have already been paid their restricted maxima. By its definition, the AL-value always selects a core allocation. For convex games (Shapley, 1971), lemacols and the marginal worth vectors coincide.

For arbitrary transferable utility (TU) games, we obtain that when a marginal worth vector is in the core, it coincides with the corresponding lemiral (the vector of lexicograhic minima over the set of coalitionally rational payoff vectors) and also with the lemaral (the vector of lexicographic maxima over the set of dual coalitionally rational payoffs) associated to the reverse order of the players. Similarly, if a lemiral / lemaral is in the core, it coincides with the corresponding lemicol / lemacol (the vector of the lexicograhically minimized / maximized payoffs over the core). The roles of the allocations in these implications are not symmetric.

\footnotetext{
1 Lemacols are named lexinals in (Tijs et al., 2011). We change the name trying to find a common nomenclature that includes the lexicographic minimization over the core and also the lexicographic minimization (respectively maximization) over the set of coalitionally rational payoff vectors (respectively over the set of dual coalitionally rational payoff vectors), where efficiency is not required.
} 
For assignment games in general, not all lemirals are in the core. ${ }^{2}$ We find, however, that if we consider the lemarals, all of them are extreme core points. To prove that, we first show that to obtain the set of payoff vectors where each coalition is paid at most its marginal contribution to the grand coalition (the dual coalitional value), it is enough to consider the marginal contributions of individuals and mixed-pairs. Moreover, these upper bounds for the core payoffs can be obtained from the initial matrix of mixed-pair valuations with no need of solving optimization problems.

The coincidence between the set of lemarals and the set of extreme core points implies that lemarals coincide with the corresponding lemacols. This provides a way of computing the AL-value of an assignment game with no need to obtain the entire coalitional function of the dual game.

The paper is organized as follows. After some general preliminaries on TU games, in Section 3 we establish some relationships between different lexicographic allocations. In Section 4 we introduce assignment games and recall known results regarding their extreme core points. We determine the essential coalitions in the dual assignment game in Section 5 and provide an algorithm for the computation of the values of the essential dual coalitions in Section 6. Finally, in Section 7 we prove that assignment games are not only ONTO-lemaral (all core extreme points are lemarals) but also INTO-lemaral games (all lemarals are core extreme points), which means that the set of extreme core allocations coincides with the set of lemarals. Section 8 concludes with a remark regarding the computation of the AL-value in assignment games.

\section{General preliminaries on games}

A transferable utility cooperative game on the nonempty finite set $N$ of players is defined by a coalitional function $w: 2^{N} \rightarrow \mathbb{R}$ satisfying $w(\varnothing)=0$. The function $w$ specifies the worth of every coalition $S \subseteq N$.

Given a game $(N, w)$, a payoff allocation $x \in \mathbb{R}^{N}$ is called efficient, if $x(N)=$ $w(N)$; individually rational, if $x_{i}=x(\{i\}) \geq w(\{i\})$ for all $i \in N$; coalitionally rational, if $x(S) \geq w(S)$ for all $S \subseteq N$; where, by the standard notation, $x(S)=\sum_{i \in S} x_{i}$ if $S \neq \varnothing$, and $x(\varnothing)=0$. We denote by $\mathcal{I}(N, w)$ the imputation set (i.e., the set of efficient and individually rational payoffs), and by $\mathcal{C}(N, w)$ the core (i.e., the set of efficient and coalitionally rational payoffs) of the game $(N, w)$. A game is balanced, if its core is not empty, and totally balanced, if every subgame is balanced. A (balanced) game is exact, if for each $S \subseteq N$ there exists a core element $x$ such that $x(S)=w(S)$. A (balanced) game has a large core, if for any coalitionally rational allocation $y$ with $y(N)>w(N)$ there exists a core element $x$ such that $x_{i} \leq y_{i}$ for all $i \in N$.

Let the collection $\mathcal{B} \subseteq 2^{N}$ contain all essential coalitions in the game (i.e. for each $S \in 2^{N} \backslash \mathcal{B}$ there is a proper partition $\left\{S_{1}, \ldots, S_{r}\right\} \subseteq \mathcal{B}, r \geq 2$, of $S$ such that $w(S) \leq \sum_{j=1}^{r} w\left(S_{j}\right)$ holds), then it is clear that the core is completely determined by such a collection $\mathcal{B}$ and the grand coalition $N$, i.e.

$$
\mathcal{C}(N, w)=\left\{x \in \mathbb{R}^{N}: x(N)=w(N), \quad x(S) \geq w(S) \forall S \in \mathcal{B}\right\} .
$$

2 Izquierdo et al. (2007) show that in an assignment game all the lemirals are extreme core points if and only if the game is exact, or alternatively, if the game has a large core. 
There are many classes of balanced games discussed extendedly in the literature (the class of assignment games is a prime example) for which the efficient computability of the core and related solutions relies on the existence of such a family $\mathcal{B}$ consisting of only polynomial many coalitions.

An order on the player set $N$ is a bijection $\sigma:\{1,2, \ldots, n\} \rightarrow N$, where for all $i \in\{1,2, \ldots, n\}, \sigma_{i}=\sigma(i)$ is the player that occupies position $i$. The set of predecessors of agent $k \in N$ in the ordering $\sigma$ is $P_{k}^{\sigma}=\left\{j \in N \mid \sigma^{-1}(j)<\sigma^{-1}(k)\right\}$. For each order $\sigma$ on the player set of game $(N, w)$, a marginal payoff vector $m^{\sigma, w}$ is defined as follows: for each $i \in\{1,2, \ldots, n\}$ let $m_{\sigma_{i}}^{w, \sigma}=w\left(P_{\sigma_{i}}^{\sigma} \cup\left\{\sigma_{i}\right\}\right)-w\left(P_{\sigma_{i}}^{\sigma}\right)$. Marginal payoff vectors are efficient, but they may not be in the core. However, if a marginal payoff vector is in the core then it is an extreme core point.

There exist in the literature other types of payoff vectors, that also sequentially allocate payoffs following a given order on the player set, but do not modify the payoffs already allocated to predecessor players. We discuss several variants of such "lexicographic" payoff vectors in this paper.

For a balanced game $(N, w)$ and an order $\sigma$ on the player set $N$, the $\sigma$-lemacol $\bar{\lambda}^{\sigma, w} \in \mathbb{R}^{N}$ is defined (Tijs et al., 2011) ${ }^{3}$ as the lexicographical maximum on $\mathcal{C}(N, w)$ with respect to $\sigma$, that is, for each $i=1,2, \ldots, n$, let

$$
\bar{\lambda}_{\sigma_{i}}^{\sigma, w}=\max \left\{x_{\sigma_{i}}: x \in \mathcal{C}(N, w), x_{\sigma_{l}}=\bar{\lambda}_{\sigma_{l}}^{\sigma, w} \text { for all } l \in\{1, \ldots, i-1\}\right\} .
$$

It is straightforward to notice that every lemacol is an extreme point of the core. On the other hand, not all extreme core points of an arbitrary balanced game need to be lemacols, as an example in (Tijs et al., 2011) or Example 5 below demonstrates.

Then, for a balanced game $(N, w)$, the Average Lexicographical value, AL-value, $A L(w)$ is defined as the average of all lemacols:

$$
A L(w)=\frac{1}{|N| !} \sum_{\sigma \in \Pi(N)} \bar{\lambda}^{\sigma, w},
$$

where $\Pi(N)$ denotes the set of orders over the player set $N$. Non-cooperative and axiomatic characterizations for the $\mathrm{AL}^{-v a l u e}{ }^{4}$ as a single-valued core selector for balanced games are given by Kongo et al. (2010).

We can also define, for each order $\sigma$ on the player set of balanced game $(N, w)$, the payoff vector of lexicographical minima over the core, the $\sigma$-lemicol $\underline{\lambda}^{\sigma, w} \in \mathbb{R}^{N}$ : for each $i=1,2, \ldots, n$, let

$$
\underline{\lambda}_{\sigma_{i}}^{\sigma, w}=\min \left\{x_{\sigma_{i}}: x \in C(N, w), x_{\sigma_{l}}=\underline{\lambda}_{\sigma_{l}}^{\sigma, w} \text { for all } l \in\{1, \ldots, i-1\}\right\} .
$$

In (Tijs et al., 2011) these payoff vectors of lexicographical minima are called reverse lexinals and their average is the reverse $A L$-value.

The lemicols can be obtained by solving sequences of linear programming problems, but, as it also happens with the lemacols, we do not have a closed (even if iterative) formula for their computation. Trying to overcome this difficulty, we

3 These lexicographical maximum vectors over the core are called lexinals in (Tijs et al., 2011) and leximals in (Kongo et al., 2010).

4 The AL-value was first introduced by Prof. Stef Tijs with the name of Alexia value. 
may not require efficiency and consider, instead of the core, the set of coalitionally rational payoff vectors denoted by

$$
\mathcal{R}(N, w)=\left\{x \in \mathbb{R}^{N}: x(S) \geq w(S) \forall S \subseteq N\right\} .
$$

We consider a simple procedure to compute 'as small as possible' coalitionally rational payoff vectors in hoping to get an efficient one, i.e. a core element. Similar to the way we get a marginal payoff vector, we compute the payoffs according to an externally given priority order of the players.

Given a game $(N, w)$, for an order $\sigma$ of the players, the $\sigma$-lemiral vector $\underline{r}^{\sigma, w} \in$ $\mathbb{R}^{N}$ is defined as follows: for each $i=1,2, \ldots, n$,

$$
\underline{r}_{\sigma_{i}}^{\sigma, w}=\min \left\{x_{\sigma_{i}}: x \in \mathcal{R}(N, w), x_{\sigma_{l}}=\underline{r}_{\sigma_{l}}^{\sigma, w} \forall l \in\{1, \ldots, i-1\}\right\},
$$

which trivially leads to

$$
\underline{r}_{\sigma_{i}}^{\sigma, w}=\max \left\{w\left(Q \cup\left\{\sigma_{i}\right\}\right)-\underline{r}^{\sigma, w}(Q): Q \subseteq P_{\sigma_{i}}^{\sigma}\right\} .
$$

It is straightforward to notice that, as it happens with the core, only essential coalitions are needed to define $\mathcal{R}(N, w)$, which means that, if $\mathcal{B}$ is a set which contains all essential coalitions for $(N, w)$, then the $\sigma$-lemiral vector is in fact

$$
\underline{r}_{\sigma_{i}}^{\sigma, w}=\max \left\{w\left(Q \cup\left\{\sigma_{i}\right\}\right)-\underline{r}^{\sigma, w}(Q): Q \subseteq P_{\sigma_{i}}^{\sigma}, Q \in \mathcal{B}\right\} .
$$

We emphasize that this simplification could provide polynomial time computability for each $\sigma$-lemiral vector for some special classes of games.

Obviously, $\underline{r}_{\sigma_{1}}^{\sigma, w}=w\left(\sigma_{1}\right)$, the minimum payoff to player $\sigma_{1}$ in any coalitionally rational payoff vector. Similarly, for any $i \in\{2, \ldots, n\}$, the amount $\underline{r}_{\sigma_{i}}^{\sigma, w}$ is the smallest possible payoff needed to satisfy all $\underline{r}^{\sigma, w}\left(Q \cup\left\{\sigma_{i}\right\}\right) \geq w\left(Q \cup\left\{\sigma_{i}\right\}\right)$ rationality inequalities for coalitions containing only $\sigma_{i}$ and his predecessors, given the already determined conditionally minimal coalitionally rational payoffs. Thus, the lemiral vector $\underline{r}^{\sigma, w}$ is the lexicographical minimum on coalitionally rational payoff vectors in $w$ with respect to $\sigma$, explaining its 'name'. In general, we will name $\sigma$-lexicographic allocations those sets of vectors that, given an order $\sigma$ on the player set, are defined following a procedure of lexicographical minima or maxima over a given set of payoff vectors.

For any position $i \in\{1, \ldots, n\}$, let $Q_{i} \subseteq P_{\sigma_{i}}^{\sigma}$ denote a maximizing coalition in (4). The lemiral $\underline{r}^{\sigma, w}$ satisfies all inequalities in the system $x \in \mathbb{R}^{N}, x\left(Q_{i} \cup\left\{\sigma_{i}\right\}\right) \geq$ $w\left(Q_{i} \cup\left\{\sigma_{i}\right\}\right), i \in\{1, \ldots, n\}$, as equalities. Since the coefficient matrix is 'triangular', hence invertible, the lemiral $\underline{r}^{\sigma, w}$ is an extreme element of the convex polyhedral set $\mathcal{R}(N, w)$. It follows that if $\underline{r}^{\sigma, w}$ is efficient then it is an extreme element of the core, $\underline{r}^{\sigma, w} \in \operatorname{ext} \mathcal{C}(N, w)$. This is another similarity to the marginal allocation procedure: if the outcome is in the core, it is an extreme point of the core.

\section{Some relationships for $\sigma$-lexicographic allocations}

We have seen so far that the aforementioned $\sigma$-lexicographical allocations present several similarities. In this section we analyze under which circumstances the different payoff vectors defined from a same order $\sigma$ do coincide. Later on, new $\sigma$-lexicographic allocations are introduced by considering the dual game. 
Moreover, we are interested in classes of games where either all extreme core payoff vectors belong to one of the defined classes of $\sigma$-lexicographic allocations, or conversely, all outcomes of a given $\sigma$-lexicographic procedure are extreme core allocations. To this end, we introduce the following definition.

A balanced game $(N, w)$ is called

- ONTO-marginal (or ONTO-lemacol, or ONTO-lemicol, or ONTO-lemiral) if all its extreme core points are marginal payoff vectors (or lemacols, or lemicols, or lemirals, respectively).

- INTO-marginal (or INTO-lemacol, or INTO-lemicol, or INTO-lemiral) if all marginal payoff vectors (or lemacols, or lemicols, or lemirals, respectively) are extreme core points of $(N, w)$.

There are some known classes of ONTO-marginal games: convex games (Shapley, 1971), information graph games (Kuipers, 1993) and assignment games (Hamers et al., 2002). Contrary to that, the class of exact games, which contains the class of convex games, is not ONTO-marginal. In fact, there are exact games where none of its extreme core points are marginal payoff vectors (see e.g. Example 1 below). Precisely the same statements can be made about another superset of convex games: the class of games with a large core.

By definition, all balanced games are INTO-lemicols and INTO-lemacols. Convex games are also INTO-marginal games (Shapley, 1971). Moreover, the property INTO-marginal characterizes the class of convex games (Ichiishi, 1981). A similar characterization of the INTO-lemiral balanced games is not known to us. We can only show that the INTO-lemiral property is necessary for largness of the core.

Proposition 1 Any balanced game with large core is INTO-lemiral.

Proof. Let $(N, w)$ be a game with large core. Notice first that for all $\sigma \in \Pi(N)$, $\underline{r}^{\sigma, w}$ is coalitionally rational. Indeed, for all $S \subseteq N$, let $i=\sigma_{k}$ be the last agent of $S$ in the order $\sigma$. Then,

$$
\underline{r}_{i}^{\sigma, w} \geq w(S \backslash\{i\} \cup\{i\})-\underline{r}^{\sigma, w}(S \backslash\{i\}),
$$

which implies $\underline{r}^{\sigma, w}(S) \geq w(S)$.

Now, if $(N, w)$ has a large core, there exists $x \in C(w)$ such that $x_{i} \leq \underline{r}_{i}^{\sigma, w}$ for all $i \in N$. But note that for all $i=\sigma_{k} \in N$, there is a coalition $Q \subseteq P_{\sigma_{k}}^{\sigma}$ such that $\underline{r}^{\sigma, w}(\{i\} \cup Q)=w(\{i\} \cup Q)$ and hence no coordinate of $\underline{r}^{\sigma, w}$ can be decreased without violating some coalitional rationality inequality. As a consequence, $x_{i}=\underline{r}_{i}^{\sigma, w}$ for all $i \in N$ and $\underline{r}^{\sigma, w}$ belongs to the core.

The examples in this paper and the strong similarities of the lemirals and the lexicographic allocations used by van Gellekom et al. (1999) to characterize largeness of the core suggest to us the coincidence of these two classes of games. Some of the results in (Estévez-Fernández, 2012) also seem to support the conjecture that the implication of Proposition 1 can be reversed.

Moreover, since convexity implies largeness of the core, we have the following hierarchy: for any game,

$$
\text { INTO-marginal } \Rightarrow \text { INTO-lemiral } \Rightarrow \text { INTO-lemicol. }
$$


The first implication follows from Proposition 1 and the second one is trivial since all balanced games are INTO-lemicol.

We now show that the property ONTO-marginal implies ONTO-lemiral and some other properties, as a consequence of the next and subsequent propositions.

Proposition 2 In a game $(N, w)$, for any ordering $\sigma$ of the players, $m^{\sigma, w} \in \mathcal{C}(N, w)$ if and only if $m^{\sigma, w}=\underline{r}^{\sigma, w}$.

Proof. If $m^{\sigma, w}=\underline{r}^{\sigma, w}$, then since $m^{\sigma, w}$ is efficient and $\underline{r}^{\sigma, w}$ is coalitionally rational, we trivially obtain that this payoff vector belongs to the core. We prove the converse implication inductively, according to the order $\sigma$. Trivially, without any condition, $m_{\sigma_{1}}^{\sigma, w}=w\left(\sigma_{1}\right)=\underline{r}_{\sigma_{1}}^{\sigma, w}$.

For arbitrary $i \in\{2, \ldots, n\}$, we assume to have $m_{\sigma_{j}}^{\sigma, w}=\underline{r}_{\sigma_{j}}^{\sigma, w}$ for all $1 \leq j \leq i-1$, implying $m^{\sigma, w}(Q)=\underline{r}^{\sigma, w}(Q)$ for all $Q \subseteq P_{\sigma_{i}}^{\sigma}$. Since $m^{\sigma, w} \in \mathcal{C}(N, w)$, we have $m_{\sigma_{i}}^{\sigma, w} \geq w\left(Q \cup\left\{\sigma_{i}\right\}\right)-m^{\sigma, w}(Q)=w\left(Q \cup\left\{\sigma_{i}\right\}\right)-\underline{r}^{\sigma, w}(Q)$ for all $Q \subset P_{\sigma_{i}}^{\sigma}$. By definition, $m_{\sigma_{i}}^{\sigma, w}=w\left(P_{\sigma_{i}}^{\sigma} \cup\left\{\sigma_{i}\right\}\right)-m^{\sigma, w}\left(P_{\sigma_{i}}^{\sigma}\right)=w\left(P_{\sigma_{i}}^{\sigma} \cup\left\{\sigma_{i}\right\}\right)-\underline{r}^{\sigma, w}\left(P_{\sigma_{i}}^{\sigma}\right)$, implying that $P_{\sigma_{i}}^{\sigma}$ is a maximizing coalition in (4). Hence, $m_{\sigma_{i}}^{\sigma, w}=\underline{r}_{\sigma_{i}}^{\sigma, w}$.

Proposition 2 implies that if for an ordering the associated marginal vector is in the core, so does the associated lemiral vector. The following example shows that the converse implication needs not hold.

Example 1 Consider the following 4-player, symmetric game:

$$
v(S)= \begin{cases}0 & \text { if }|S| \leq 1 \\ 3 & \text { if }|S|=2 \\ 5 & \text { if }|S|=3 \\ 10 & \text { if }|S|=4\end{cases}
$$

It is easily checked that for the order $\sigma=(1,2,3,4)$, the lemiral vector $\underline{r}^{\sigma, v}=$ $(0,3,3,4)$ is a core element, but the associated marginal vector $m^{\sigma, v}=(0,3,2,5)$ is not. In fact, none of the marginal vectors (that are the permutations of $(0,3,2,5))$ is in the core, but all the lemirals are. Indeed, for any order, the lemiral payoff to the first player is 0 , to the second and to the third player it is 3 , and to the last player the payoff is 4 . Thus, for all orders, the associated lemiral vector is an (extreme) element of the core, i.e. this is an INTO-lemiral game. The core, however, has other vertices, e.g. the vector $(1,2,2,5)$ and all of its permuted variants are also extreme elements of the core, i.e. this game is not ONTO-lemiral.

Observe that the family of extreme core vectors $(0,3,3,4),(1,2,2,5)$ and their permuted variants make all coalitional rationality inequalities tight, so this game is an exact game. And it also has a large core, since for totally balanced symmetric games both notions are equivalent (see Biswas et al., 1999). Hence Proposition 1 could have been applied to deduce that all lemirals belong to the core.

The lemiral and the lemicol vectors are determined on two different - although for balanced games closely related - sets of payoffs. In general, already the first player in an order can receive different payoffs in the lemiral and in the lemicol allocations. The following proposition states when requiring efficiency of the allocation makes no difference. 
Proposition 3 In a game $(N, w)$ for any ordering $\sigma$ of the players, $\underline{r}^{\sigma, w} \in \mathcal{C}(N, w)$ if and only if $\underline{\lambda}^{\sigma, w}=\underline{r}^{\sigma, w}$.

Proof. The lemicol vectors are in the core by definition, so the "if" direction needs no explanation.

We show the "only if" direction inductively, according to the order $\sigma$. Trivially, $\underline{\lambda}_{\sigma_{1}}^{\sigma, w} \geq \underline{r}_{\sigma_{1}}^{\sigma, w}=w\left(\sigma_{1}\right)$. Now, if $\underline{r}^{\sigma, w} \in \mathcal{C}(N, w)$ then the core minimum payoff to player $\sigma_{1}$ must be $\underline{r}_{\sigma 1}^{\sigma, w}$.

For arbitrary $i \in\{2, \ldots, n\}$, we assume to have $\underline{\lambda}_{\sigma_{j}}^{\sigma, w}=\underline{r}_{\sigma_{j}}^{\sigma, w}$ for all $1 \leq j \leq i-1$. Then by comparing the minimization problems in (2) and (3), we get $\underline{\lambda}_{\sigma_{i}}^{\sigma, w} \geq \underline{r}_{\sigma_{i}}^{\sigma, w}$. Here again, $\underline{r}^{\sigma, w} \in \mathcal{C}(N, w)$ implies that the conditional core minimum payoff to player $\sigma_{i}$ must be $\underline{r}_{\sigma_{i}}^{\sigma, w}$, concluding the inductive step.

Applied to the game in Example 1, Proposition 3 implies that the set of lemicol vectors consists of the vector $(0,3,3,4)$ and its permuted variants. In this example, all lemirals belong to the core, but this needs not always be the case, as the next example shows. It also demonstrates that the roles of lemirals and lemicols in Proposition 3 are not interchangeable.

Example 2 Consider the following 4-player, symmetric game:

$$
v(S)= \begin{cases}0 & \text { if }|S| \leq 1 \\ 4 & \text { if }|S|=2 \\ 5 & \text { if }|S|=3 \\ 8 & \text { if }|S|=4\end{cases}
$$

The core of this game is a singleton, $C(N, v)=\{(2,2,2,2)\}$. If we take the order $\sigma=(1,2,3,4)$ and compute the corresponding lemiral, we find $\underline{r}^{\sigma, v}=(0,4,4,4)$, which is not in the core.

The combination of the two above propositions leads to a similar connection between marginal payoff vectors and lemicols.

Corollary 1 In a game $(N, w)$, for any ordering $\sigma$ of the players, $m^{\sigma, w} \in \mathcal{C}(N, w)$ if and only if $m^{\sigma, w}=\underline{\lambda}^{\sigma, w}$.

Proof: The "if" part is obvious since lemicols belong to the core by definition. For the "only if" part, notice that if $m^{\sigma, w} \in \mathcal{C}(N, w)$, Proposition 2 implies $m^{\sigma, w}=\underline{r}^{\sigma, w}$ and hence $\underline{r}^{\sigma, w} \in \mathcal{C}(N, w)$. Then, Proposition 3 implies $m^{\sigma, w}=\underline{r}^{\sigma, w}=\underline{\lambda}^{\sigma, w}$.

In the above corollary the roles of the marginal payoff vector and that of the lemicol are not interchangeable. The lemicols always belong to the core by definition, while it is easy to find examples where some marginal payoff vector is not a core allocation. Take for instance Example 1 where none of the marginal payoff vectors belongs to the core.

The following hierarchy of the properties summarizes our results presented so far: for any game

$$
\text { ONTO-marginal } \Rightarrow \text { ONTO-lemiral } \Rightarrow \text { ONTO-lemicol. }
$$


As a consequence, for convex games, information graph games and assignment games, all extreme core allocations are not only marginal payoff vectors but also lemirals and lemicols.

Notice that the 4-player, exact, symmetric game in Example 1 is not ONTOlemicol. Indeed, the extreme core point $(1,2,2,5)$ is not a lemicol since the first player in any order should get 0 . However, $(1,2,2,5)$ is the lemacol related to the reverse order $\sigma=(4,3,2,1)$ of the players. In order to look for new relationships between the lemacols and other lexicographic allocations we need to introduce some new notions.

Since $\mathcal{R}(N, w)$ is not bounded above, we do not propose a lexicographic maximization over this set of coalitionally rational payoffs. We now define a set of payoff vectors supported by a similar "rationality" requirement: instead of imposing that no coalition receives less than its coalitional value, we require that no coalition receives more than its dual coalitional value, i.e. its contribution to the grand coalition. Given a game $(N, w)$, we name this set of payoffs the set of dual coalitionally rational payoffs and write

$$
\mathcal{R}^{*}(N, w)=\left\{x \in \mathbb{R}^{N}: x(S) \leq w(N)-w(N \backslash S) \text { for all } S \subseteq N\right\} .
$$

Given a game $(N, w)$, the game $\left(N, w^{*}\right)$ defined by $w^{*}(S)=w(N)-w(N \backslash S)$ for all $S \subseteq N$ is known in the literature as the dual game and thus inspires our definition of the dual coalitionally rational payoffs. Notice that $w^{*}(\varnothing)=0$ and $w^{*}(N)=w(N)$ for any game $(N, w)$. It follows that, under efficiency, the sets of coalitionally rational payoffs and of dual coalitionally rational payoffs coincide and also coincide with the core. In other words, the core of any coalitional game coincides with the anticore of its dual game, that is,

$$
\mathcal{C}(N, w)=\mathcal{C}^{*}\left(N, w^{*}\right):=\left\{x \in \mathbb{R}^{N}: x(N)=w^{*}(N), x(S) \leq w^{*}(S) \forall S \subseteq N\right\} .
$$

As it happens with the core and the set of coalitionally rational payoff vectors, for certain games, some coalitions may be redundant in the definition of the anticore and of the dual coalitionally rational payoff set. A coalition $S$ is called inessential in the dual of a game $(N, w)$ if it has a proper partition in non-empty coalitions $S_{1}, \ldots, S_{k}, k \geq 2$, such that $w^{*}(S) \geq w^{*}\left(S_{1}\right)+\ldots+w^{*}\left(S_{k}\right)$. Notice that if a coalition is inessential in the dual game, it is redundant for the set of dual coalitionally rational payoff vectors and for the anticore. Then the analogous statement to (1) easily follows: let the collection $\mathcal{D} \subseteq 2^{N}$ contain all essential coalitions in the dual game $\left(N, w^{*}\right)$, then the core of the game is completely determined by efficiency and such a collection $\mathcal{D}$, i.e.

$$
\mathcal{C}(N, w)=\mathcal{C}^{*}\left(N, w^{*}\right)=\left\{x \in \mathbb{R}^{N}: x(N)=w^{*}(N), x(T) \leq w^{*}(T) \forall T \in \mathcal{D}\right\} .
$$

In the second part of this paper, we identify such a family $\mathcal{D}$ consisting of quadratic many coalitions for assignment games.

Over the set $\mathcal{R}^{*}(N, w)$ of dual coalitionally rational payoff vectors, we propose a lexicographic maximization procedure. Given a game $(N, w)$, for an order $\sigma$ of the players, the $\sigma$-lemaral vector $\bar{r}^{\sigma, w} \in \mathbb{R}^{N}$ is defined as follows: for all $i \in\{1,2, \ldots, n\}$,

$$
\bar{r}_{\sigma_{i}}^{\sigma, w}=\max \left\{x_{\sigma_{i}}: x \in \mathcal{R}^{*}(N, w), x_{\sigma_{l}}=\bar{r}_{\sigma_{l}}^{\sigma, w} \forall l \in\{1, \ldots, i-1\}\right\},
$$


which trivially leads to

$$
\bar{r}_{\sigma_{i}}^{\sigma, w}=\min \left\{w^{*}\left(Q \cup\left\{\sigma_{i}\right\}\right)-\bar{r}^{\sigma, w}(Q): Q \subseteq P_{\sigma_{i}}^{\sigma}\right\} .
$$

The counterpart of statement (5) is as follows: if the collection $\mathcal{D} \subseteq 2^{N}$ contains all essential coalitions in the dual game $\left(N, w^{*}\right)$, then

$$
\bar{r}_{\sigma_{i}}^{\sigma, w}=\min \left\{w^{*}\left(Q \cup\left\{\sigma_{i}\right\}\right)-\bar{r}^{\sigma, w}(Q): Q \subseteq P_{\sigma_{i}}^{\sigma}, Q \in \mathcal{D}\right\} .
$$

As we will see below, for assignment games this reduction could provide polynomial time computability for each $\sigma$-lemaral vector.

Now that all the lexicographic allocations we need have been introduced, we provide a summary table for our nomenclature: the rows identify the type of lexicographic optimization and the columns specify the domain.

\begin{tabular}{c||c|c|c|} 
& Co & $\mathbf{R a}$ & $\mathbf{R a}^{*}$ \\
\hline \hline $\min$ & lemicol & lemiral & - \\
\hline $\max$ & lemacol & - & lemaral \\
\hline
\end{tabular}

The next proposition is the counterpart of Proposition 2 as it states that ONTO-marginal also implies ONTO-lemaral. Given any order $\sigma \in \Pi(N)$, let us define the reverse order $\sigma^{*} \in \Pi(N)$ by $\sigma_{i}^{*}=\sigma_{n-i+1}$ for all $i \in\{1,2, \ldots, n\}$.

Proposition 4 In a game $(N, w)$, for any ordering $\sigma$ of the players, $m^{\sigma, w} \in \mathcal{C}(N, w)$ if and only if $m^{\sigma, w}=\bar{r}^{\sigma^{*}, w}$.

Proof. The "if" part is obvious since whenever a lemaral is efficient it belongs to the core.

Let us prove the "only if" implication. Assume that for some $\sigma \in \Pi(N), m^{\sigma, w} \in$ $\mathcal{C}(N, w)$. Observe first of all that since the marginal vector satisfies $m^{\sigma, w}\left(\left\{\sigma_{1}, \ldots, \sigma_{n-k}\right\}\right)$ $=w\left(\left\{\sigma_{1}, \ldots, \sigma_{n-k}\right\}\right)$ for all $0 \leq k \leq n-1$, it also satisfies

$$
m^{\sigma, w}\left(\left\{\sigma_{1}^{*}, \ldots, \sigma_{k}^{*}\right\}\right)=w^{*}\left(\left\{\sigma_{1}^{*}, \ldots, \sigma_{k}^{*}\right\}\right) \quad \text { for all } 1 \leq k \leq n .
$$

Then, trivially from $(7)$, without any condition, $m_{\sigma_{1}^{*}}^{\sigma, w}=w^{*}\left(\left\{\sigma_{1}^{*}\right\}\right)=\bar{r}_{\sigma_{1}^{*}}^{\sigma^{*}, w}$.

For arbitrary $i \in\{2, \ldots, n\}$, we assume to have $m_{\sigma_{j}^{*}}^{\sigma, w}=\bar{r}_{\sigma_{j}^{*}}^{\sigma^{*}, w}$ for all $1 \leq j \leq i-1$, implying $m^{\sigma, w}(Q)=\bar{r}^{\sigma^{*}, w}(Q)$ for all $Q \subseteq P_{\sigma_{i}^{*}}^{\sigma^{*}}$. Since $m^{\sigma, w} \in \mathcal{C}(N, w)$, we have $m_{\sigma_{i}^{*}}^{\sigma, w} \leq w^{*}\left(Q \cup\left\{\sigma_{i}^{*}\right\}\right)-m^{\sigma, w}(Q)=w^{*}\left(Q \cup\left\{\sigma_{i}^{*}\right\}\right)-\bar{r}^{\sigma^{*}, w}(Q)$ for all $Q \subset P_{\sigma_{i}^{*}}^{\sigma^{*}}$. It follows from (9) and the inductive assumption that $m_{\sigma_{i}^{*}}^{\sigma, w}=w^{*}\left(P_{\sigma_{i}^{*}}^{\sigma^{*}} \cup\left\{\sigma_{i}^{*}\right\}\right)-m^{\sigma, w}\left(P_{\sigma_{i}^{*}}^{\sigma^{*}}\right)=$ $w^{*}\left(P_{\sigma_{i}^{*}}^{\sigma^{*}} \cup\left\{\sigma_{i}^{*}\right\}\right)-\bar{r}^{\sigma^{*}, w}\left(P_{\sigma_{i}^{*}}^{\sigma^{*}}\right)$, implying that $P_{\sigma_{i}^{*}}^{\sigma^{*}}$ is a minimizing coalition in (7). Hence, $m_{\sigma_{i}^{*}}^{\sigma, w}=\bar{r}_{\sigma_{i}^{*}}^{\sigma^{*}, w}$.

Notice that the roles of lemarals and marginals in the above proposition are not interchangeable. For instance, in Example 1, the lemaral corresponding to order $\sigma=(1,2,3,4)$ is $\bar{r}^{\sigma, w}=(5,2,2,1)$, which belongs to the core. In fact all lemarals are obtained by permuting the components of this payoff vector. However, recall that for this game no marginal payoff vector is in the core.

We now prove the counterpart of Proposition 3, namely that property ONTOlemaral implies ONTO-lemacol. 
Proposition 5 In a game $(N, w)$, for any ordering $\sigma$ of the players, $\bar{r}^{\sigma, w} \in \mathcal{C}(N, w)$ if and only if $\bar{\lambda}^{\sigma, w}=\bar{r}^{\sigma, w}$.

Proof. Since all lemacol payoff vectors are core elements by definition, whenever a lemacol equals a lemaral, this lemaral payoff vector belongs to the core. To see the converse statement, notice first that $\bar{r}_{\sigma_{1}}^{\sigma, w}=w^{*}\left(\left\{\sigma_{1}\right\}\right)=\bar{\lambda}_{\sigma_{1}}^{\sigma, w}$. Assume now that for all $1 \leq j \leq i-1$ it holds $\bar{r}_{\sigma_{j}}^{\sigma, w}=\bar{\lambda}_{\sigma_{j}}^{\sigma, w}$. Then,

$$
\bar{r}_{\sigma_{i}}^{\sigma, w}=\min \left\{w^{*}\left(Q \cup\left\{\sigma_{i}\right\}\right)-\bar{r}^{\sigma, w}(Q): Q \subseteq P_{\sigma_{i}}^{\sigma}\right\} \geq \bar{\lambda}_{\sigma_{i}}^{\sigma, w},
$$

where the inequality follows since $\bar{\lambda}^{\sigma, w} \in \mathcal{C}(N, w)$. On the other hand, since we assume $\bar{r}^{\sigma, w} \in \mathcal{C}(N, w)$, the definition of $\bar{\lambda}^{\sigma, w}$ and the fact that $\bar{r}_{\sigma_{j}}^{\sigma, w}=\bar{\lambda}_{\sigma_{j}}^{\sigma, w}$ for all $1 \leq j \leq i-1$, implies the converse inequality $\bar{r}_{\sigma_{i}}^{\sigma, w} \leq \bar{\lambda}_{\sigma_{i}}^{\sigma, w}$. Hence we get $\bar{r}_{\sigma_{i}}^{\sigma, w}=\bar{\lambda}_{\sigma_{i}}^{\sigma, w}$.

To see that the roles of lemarals and lemacols cannot be interchanged in the above proposition, consider the dual of the game in Example 2:

$$
v^{*}(S)= \begin{cases}0 & \text { if }|S|=0 \\ 3 & \text { if }|S|=1 \\ 4 & \text { if }|S|=2 \\ 8 & \text { if }|S| \geq 3\end{cases}
$$

Given the order $\sigma=(1,2,3,4)$, the corresponding lemaral payoff vector is $\bar{r}^{\sigma, v}=$ $(3,1,1,1)$ which is not a core element. Nevertheless, by definition, all lemacols belong to the core.

To get the counterpart of Corollary 2, we combine the two last propositions to show that ONTO-marginal also implies ONTO-lemacol.

Corollary 2 In a game $(N, w)$ for any ordering $\sigma$ of the players, $m^{\sigma, w} \in \mathcal{C}(N, w)$ if and only if $m^{\sigma, w}=\bar{\lambda}^{\sigma^{*}, w}$.

Proof. If for some $\sigma, m^{\sigma, w} \in \mathcal{C}(N, w)$, Proposition 4 implies that $m^{\sigma, w}=\bar{r}^{\sigma^{*}, w} \in$ $\mathcal{C}(N, w)$. Now, Proposition ?? guarantees that $\bar{r}^{\sigma^{*}, w}=\bar{\lambda}^{\sigma *, w}$ and hence $m^{\sigma, w}=$ $\bar{\lambda}^{\sigma^{*}, w}$. The converse implication is straightforward.

We finish this section by highlighting the hierarchy of the properties related to the lexicographic maximization allocations: for any game

$$
\text { ONTO-marginal } \Rightarrow \text { ONTO-lemaral } \Rightarrow \text { ONTO-lemacol. }
$$

As a consequence, for convex games, information graph games and assignment games, all extreme core allocations are not only marginal payoff vectors but also lemarals and lemacols.

\section{Assignment games}

From now on we focus on a particular class of balanced games, namely on the class of assignment games. Taken into account that assignment games are also ONTOmarginal (Hamers et al., 2002), we get from the general implications proved in Propositions 1-4 that assignment games are ONTO-lemiral/lemaral/lemicol/lemacol. 
Since any balanced game is INTO-lemicol/lemacol by definition, we will focus on the INTO-lemiral/lemaral properties for assignment games. We first recall some known related results in this section.

Given two disjoint finite sets $S$ and $T$, we call $\mu \subseteq S \times T$ an $(S, T)$-assignment, if it is a bijection from some $S^{\prime} \subseteq S$ to some $T^{\prime} \subseteq T$ such that $\left|S^{\prime}\right|=\left|T^{\prime}\right|=\min (|S|,|T|)$. Trivially, $\mu=\varnothing$, if $S=\varnothing$ or $T=\varnothing$. We shall write $(i, j) \in \mu$ as well as $\mu(i)=j$. We denote by $\mathcal{M}(S, T)$ the set of all $(S, T)$-assignments. Obviously, $\mathcal{M}(S, T)=\{\varnothing\}$, if $S=\varnothing$ or $T=\varnothing$.

A game $(N, w)$ is called an assignment game, if there exists a partition $N=I \cup J$, $I \cap J=\varnothing$, of the player set and a non-negative matrix $A=\left[a_{i j}\right]_{i \in I, j \in J}$ such that

$$
w(S)=w_{A}(S):=\max _{\mu \in \mathcal{M}(S \cap I, S \cap J)} \sum_{(i, j) \in \mu} a_{i j} \quad \text { for all } S \subseteq N .
$$

A matching $\mu \in \mathcal{M}(S \cap I, S \cap J)$ such that $w_{A}(S)=\sum_{(i, j) \in \mu} a_{i j}$ is an optimal matching. Due to the non-negativity of $A$, we can (and will) assume w.l.o.g. that any optimal matching is a complete matching for the 'short side' of the coalition. We denote by $\mathcal{M}_{A}^{*}(S \cap I, S \cap J)$ the set of optimal matchings for coalition $S$. A player in $I$ or $J$ is called a row or column player, respectively. Coalitions containing one row and one column player are called mixed-pair coalitions. It is clear that

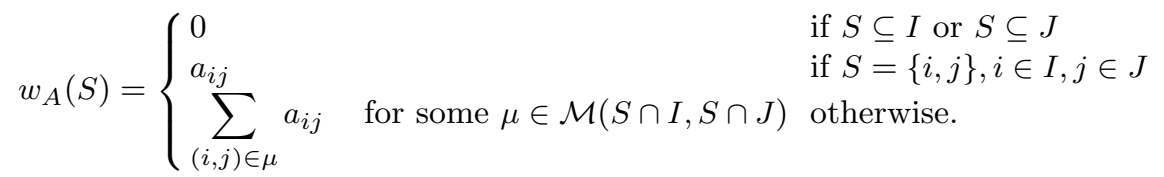

It follows that the collection

$$
\mathcal{B}=\{\{i\}: i \in I\} \cup\{\{j\}: j \in J\} \cup\{\{i, j\}: i \in I, j \in J\}
$$

contains all essential coalitions in any assignment game with player set $I \cup J$, irrespective of the matrix $A$. Shapley and Shubik (1972) proved that with the collection $\mathcal{B}$ given in (10) the simplified description (1) of the core of any assignment game is nonempty, hence assignment games are always balanced.

First, by adding dummy player(s) (i.e. zero rows/columns to the matrix $A$ ), we can assume without loss of generality that there are the same number of players of both types (i.e. the underlying data matrix $A$ is square). It is well known that the core has the dummy-player property, consequently at any core allocation of the augmented assignment game, all dummy players receive their individual values of 0 .

In order to obtain a unified notation, we introduce a fictitious row player and a fictitious column player, and consider a single-player coalition as a fictitious mixedpair coalition consisting of the 'real' player and the fictitious one of the other type. Moreover, we identify the mixed-pair coalitions with the ordered pairs of the two players, always the row player written first. More formally, $(i, j)$ denotes the 'real' mixed-pair coalition $\{i, j\}, i \in I, j \in J$; we write $(i, 0)$ for single-player coalition $\{i\}, i \in I$, and $(0, j)$ for $\{j\}, j \in J$; finally, $(0,0)$ denotes the coalition of the two fictitious players. To capture the relevant part of the original coalitional function $w_{A}$ needed for the simplified description (1) of its core, we augment the original (square) data matrix with entries $a_{i 0}=0$ for all $i \in I$, also $a_{0 j}=0$ for all $j \in J$, 
finally $a_{00}=0$. Since the type of the players is determined by their positions in the ordered pairs, it will be convenient to use a common set $M_{0}=\{0,1,2, \ldots, m\}$ of indices, where $m=|I|=|J|$.

In what remains of the paper, we assume that the rows and columns of the augmented (square) data matrix are arranged such that the diagonal assignment $\left\{(i, i): i \in M_{0}\right\}$ is of maximum value, i.e. $w_{A}(I \cup J)=\sum_{i=1}^{m} a_{i i}$, because, by definition, $a_{00}=0$.

To emphasize the bipartite nature of assignment games, we shall write the payoff allocations as $(u, v) \in \mathbb{R}^{M_{0}} \times \mathbb{R}^{M_{0}}$, but we always require $u_{0}=v_{0}=0$ to hold.

With all the above conventions, the core of the assignment game $\left(N, w_{A}\right)$ induced by matrix $A$ is

$\mathcal{C}\left(N, w_{A}\right)=\left\{(u, v): u_{0}=v_{0}=0, u_{i}+v_{i}=a_{i i} \forall i \in M_{0}, \quad u_{i}+v_{j} \geq a_{i j} \forall i \neq j \in M_{0}\right\}$.

Subclasses of assignment games were identified by properties of the underlying matrices by Solymosi and Raghavan (2001) who proved that an assignment game is exact if and only if it has a large core. Moreover, if the underlying $A$ is a square matrix with an optimal matching placed on the diagonal then both of these game properties are equivalent to the matrix property:

$$
a_{i j}+a_{k k} \geq a_{i k}+a_{k j} \quad \text { for all } i, j, k \in M_{0},
$$

that can be checked efficiently.

Izquierdo et al. (2007) defined the so-called max-payoff vectors for assignment games, and proved that

- in any assignment game all core extreme points are max-payoff vectors;

- all max-payoff vectors are extreme points of the core if and only if the assignment game is exact (equivalently, has a large core).

It is straightforward to note that the max-payoff vectors are precisely the lemiral vectors specialized for assignment games (only the single-player and the mixedpair coalitions are considered, but the underlying idea of satisfying the rationality constraints with lexicographically minimum payoffs is the same). Hence, in our terminology, Izquierdo et al. (2007) proved that

- all assignment games are ONTO-lemiral, (this can also be deduced from our Proposition 2 by taking into account that assignment games are ONTOmarginal (Hamers et al., 2002)),

- an assignment game is INTO-lemiral if and only if it is exact (equivalently, has a large core).

It is interesting to compare these results with Example 1 in the previous section, where an exact symmetric game is also INTO-lemiral but not ONTO-lemiral.

Notice that among the above discussed $\sigma$-lexicographic allocations the only ones that satisfy both the ONTO and INTO properties for all assignment games, are the lemacols and the lemicols. These allocations, however, are not easy to compute, mainly due to the pairwise efficiency equalities required for core vectors. The lemiral (max-payoff) vectors are easier to compute but they are guaranteed to be in the core only under the exactness condition (12). In the next sections we focus on the lemaral vectors and prove that these also easier-to-compute allocations characterize the extreme points of the core in all assignment games. 


\section{The dual assignment game}

It is well known that the core of any coalitional game coincides with the anticore of its dual game (cf. (6)). For assignment games, Martínez-de-Albéniz et al. (2011) showed that in the usual description (11) of the core the lower bound inequalities $u_{i}+v_{j} \geq a_{i j}=w_{A}((i, j))$ related to $i \neq j$ mixed pairs can be replaced with the dual upper bound inequalities $u_{i}+v_{j} \leq w_{A}^{*}((i, j))$, while maintaining efficiency for the grand coalition and non-negativity for the individual payoffs. Since obviously $w_{A}^{*}((i, i))=a_{i i}$ for all $i \in M$, and $w_{A}^{*}(N)=\sum_{i=1}^{m} a_{i i}$, it easily follows that the grand efficiency constraint $u(I)+v(J)=w_{A}(N)=w_{A}^{*}(N)$ can also be replaced by the pairwise efficiency equalities $u_{i}+v_{i}=w_{A}^{*}((i, i))=a_{i i}$ for all $i \in M$. It is not difficult to see (for brevity, however, we omit the details) that under efficiency this dualization process can be completed by also replacing the non-negativity restrictions for the individual payoffs with the individual dual constraints $u_{i} \leq$ $w_{A}^{*}((i, 0)) \forall i \in M$ and $v_{j} \leq w_{A}^{*}((0, j)) \forall j \in M$, and thus obtain the purely dual counterpart of (11).

$\mathcal{C}\left(w_{A}\right)=\left\{(u, v): u_{0}=v_{0}=0, u_{i}+v_{i}=w_{A}^{*}((i, i)) \forall i \in M_{0}, \quad u_{i}+v_{j} \leq w_{A}^{*}((i, j)) \forall i \neq j \in M_{0}\right\}$.

Without the efficiency constraint(s), however, it is not at all obvious whether the larger coalitions could still be omitted and such a small size description (using only quadratic many constraints) could also be obtained for the set of dual coalitionally rational payoffs in assignment games.

Interestingly, all the aformentioned core upper bounds are known to be tight. Both Demange (1982) and Leonard (1983) proved that all individual contributions to the grand coalition are attained in the core of the assignment game. Moreover, Núñez and Rafels (2002) showed that for each 'real' mixed pair $(i, j) \in I \times J$ the upper bound $w_{A}(N)-w_{A}(N \backslash\{i, j\})=w_{A}^{*}((i, j))$ of their total payoff is also attained in the core. This is a major difference with the usual individual or mixedpair lower bounds $u_{i}+v_{j} \geq a_{i j}$ for $(i, j) \in M_{0} \times M_{0}$, for some of these may not be attained in the core. Precisely the unconditional tightness of these dual upper bounds suggests that maybe the set of lemarals coincides with the set of extreme core allocations, with no need of requiring exactness as we must do for the lemirals. To give an affirmative answer to this question, we prove first that in the definition of the set of dual coalitionally rational payoff vectors of the assignment game only individual and mixed-pair coalitions are needed.

Theorem 1 Let $\left(N, w_{A}\right)$ be an assignment game. For all $S \subseteq I \cup J$ there exists a partition of $S$ in $S_{1}, S_{2}, \ldots, S_{r} \in \mathcal{B}$ such that

$$
w^{*}(S) \geq \sum_{k=1}^{r} w^{*}\left(S_{k}\right),
$$

where collection $\mathcal{B}$ is defined in (10).

Proof. Let $A$ be square and assume also without loss of generality that $|S \cap I| \geq$ $|S \cap J|$, otherwise interchange the roles of $i$ and $j$ in this proof. Let $\mu$ be an optimal (complete) matching for the grand coalition, $\mu \in \mathcal{M}_{A}^{*}(I, J)$, and let $\mu^{\prime}$ be an optimal matching for the complement $T:=N \backslash S$ of coalition $S$ (complete for its short side $T \cap I)$, that is $\mu^{\prime} \in \mathcal{M}_{A}^{*}(T \cap I, T \cap J)$. We first present two remarks. 
If there is some $i \in S \cap I$ with $\mu(i) \in S$ or $j \in S \cap J$ with $\mu^{-1}(j) \in S$, denote by $\tilde{S}$ the subset of $S$ with this property, i.e.

$$
\tilde{S}=\{k \in S \text { matched by } \mu \text { to another agent in } S\}
$$

and define $N^{\prime}=N \backslash \tilde{S}$ and $S^{\prime}=S \backslash \tilde{S}$. Then,

$$
w_{A}^{*}(S)=w_{A}(N)-w_{A}(N \backslash S)=\sum_{k \in \tilde{S} \cap I} a_{k \mu(k)}+w_{A}\left(N^{\prime}\right)-w_{A}\left(N^{\prime} \backslash S^{\prime}\right) .
$$

Since for all $k \in \tilde{S} \cap I, a_{k \mu(k)}=w_{A}(N)-w_{A}(N \backslash\{k, \mu(k)\})=w_{A}^{*}(\{k, \mu(k)\})$, equation (14) means that we can restrict our attention to the case where for all $k \in S \cap I, \mu(k) \notin S$, in fact, $\mu(k) \in T \cap J$.

Similarly, if $\mu(i)=\mu^{\prime}(i)$ holds for some $i \in I \backslash S=T \cap I$, or $\mu^{-1}(j)=\mu^{\prime-1}(j)$ holds for some $j \in J \backslash S=T \cap J$, we denote by $\hat{S}$ the set of agents with this property, i.e.

$$
\hat{S}=\left\{k \in T=N \backslash S \text { with the same partner by } \mu \text { and } \mu^{\prime}\right\} .
$$

Then,

$$
\begin{aligned}
w^{*}(S) & =w(N)-w(N \backslash S) \\
& =\sum_{i \in \hat{S} \cap I} a_{i \mu(i)}+w(N \backslash \hat{S})-\left[\sum_{i \in \hat{S} \cap I} a_{i \mu(i)}+w((N \backslash \hat{S}) \backslash S)\right] \\
& =w(N \backslash \hat{S})-w((N \backslash \hat{S}) \backslash S) .
\end{aligned}
$$

So, again, we can assume without loss of generality that for all $k \in N \backslash S$, player $k$ has different partners in $\mu$ and in $\mu^{\prime}$.

After the above two remarks, we define a directed graph $G$ with set of nodes $N$ and the following edges: if $i \in I$ and $j \in J$, there is an edge of the type $i \longrightarrow j$ if $(i, j) \in \mu$, and there is an edge $j \longrightarrow i$ whenever $(i, j) \in \mu^{\prime}$. Notice that at each node $i \in I$ we have exactly one outgoing edge and at most one incoming edge, whereas at each node $j \in J$ there is at most one outgoing edge and exactly one incoming edge. Hence, the graph $G$ is partitioned in connected components. We can assume without loss of generality that each component of $G$ is a path, since in case of a cycle $\left(i_{1}, j_{1}, i_{2}, j_{2}, \ldots, i_{k}, j_{k}, i_{1}\right)$ with $i_{h} \in I$ and $j_{h} \in J$ for all $1 \leq h \leq k$, the restrictions of $\mu$ and of $\mu^{\prime}$ to the coalition $R=\left\{i_{1}, j_{1}, i_{2}, j_{2}, \ldots, i_{k}, j_{k}\right\}$ are both optimal matchings for $R \subseteq T$, so we could alter $\mu^{\prime}$ on $R$ to coincide with $\mu$ and apply our second remark.

For each $i \in S \cap I$, let $C_{i}=\left(i=i_{1}, j_{1}, i_{2}, j_{2}, \ldots, i_{k}, j_{k}\right)$ be the maximal path in $G$ starting at $i$. Clearly, nodes in $S \cap I$ can only be starting points of paths in $G$, since they cannot have an incoming edge. Notice that such a path ends either at $j_{k} \in S$ or at $j_{k} \in J \backslash S$ not matched by $\mu^{\prime}$. Notice also that all $i_{l}$ in this path, $l \in\{2, \ldots, k\}$, belong to $I \backslash S$. Define now the following matching for $\left(I \backslash\left\{i_{1}\right\}\right) \cup\left(J \backslash\left\{j_{k}\right\}\right)$ :

$$
\tilde{\mu}=\bigcup_{i \in I \backslash C_{i_{1}}}\{(i, \mu(i))\} \cup \bigcup_{l=1}^{k-1}\left\{\left(i_{l+1}, j_{l}\right)\right\}
$$


In the first case, that is if $j_{k} \in S$, we get

$$
\begin{aligned}
w_{A}^{*}\left(\left(i_{1}, j_{k}\right)\right) & =w_{A}(N)-w_{A}\left(N \backslash\left\{i_{1}, j_{k}\right\}\right) \leq \sum_{(i, j) \in \mu} a_{i j}-\sum_{(i, j) \in \tilde{\mu}} a_{i j} \\
& =\sum_{l=1}^{k} a_{i_{l} \mu\left(i_{l}\right)}-\sum_{l=1}^{k-1} a_{i_{l+1} j_{l}}=\sum_{l=1}^{k} a_{i_{l} \mu\left(i_{l}\right)}-\sum_{l=1}^{k-1} a_{i_{l+1} \mu^{\prime}\left(i_{l+1}\right)} .
\end{aligned}
$$

In the second case, that is if $j_{k} \in J \backslash S$ but unmatched by $\mu^{\prime}$, we also get

$$
\begin{aligned}
w_{A}^{*}\left(\left(i_{1}, 0\right)\right) & =w_{A}(N)-w_{A}\left(N \backslash\left\{i_{1}\right\}\right) \leq \sum_{(i, j) \in \mu} a_{i j}-\sum_{(i, j) \in \tilde{\mu}} a_{i j} \\
& =\sum_{l=1}^{k} a_{i_{l} \mu\left(i_{l}\right)}-\sum_{l=1}^{k-1} a_{i_{l+1} \mu^{\prime}\left(i_{l+1}\right)}
\end{aligned}
$$

We now show that all pairs $(i, j) \in \mu$ and $(i, j) \in \mu^{\prime}$ belong to some component $C_{i^{\prime}}$ of $G$ for some $i^{\prime} \in S$. Notice first that if $j \in J \backslash S$, then either $\mu^{-1}(j)=i \in S$ and then the edge $(i, j)$ belongs to the component $C_{i}$; or $\mu^{-1}(j)=i_{1} \in I \backslash S$ and then, since $|J \backslash S| \geq|I \backslash S|, i_{1}$ is matched by $\mu^{\prime}$, and we denote $\mu\left(i_{1}\right)=j_{2} \in J \backslash S$. Going backwards we built a chain $\left(j_{r+1}, i_{r}, j_{r}, \ldots, i_{1}, j_{1}=j\right)$ with all $j_{l} \in J \backslash S$, for all $l \in\{1, \ldots, r+1\}$, such that $\mu^{-1}\left(j_{r+1}\right)=i_{r+1} \in S$. Then, the edge $(i, j)$ belongs to the component $C_{i_{r+1}}$. Once we have that all $(i, j) \in \mu$ with $j \in J \backslash S$ belong to some component $C_{i^{\prime}}$ with $i^{\prime} \in S$, it follows immediately that the edge $\left(\mu^{\prime-1}(j), j\right)$ also belongs to $C_{i^{\prime}}$. Hence, all pairs $(i, j) \in \mu^{\prime}$ also belong to some component $C_{i^{\prime}}$. Finally, if we have a pair $(i, j) \in \mu$ with $j \in S$, by our initial assumption we can guarantee that $i \in I \backslash S$ and then the pair $\left(i, \mu^{\prime}(i)\right)$ belongs to some component $C_{i^{\prime}}$ for $i^{\prime} \in S$. By the construction of the graph, this implies that the path that reaches $i$ can be continued with the edge $(i, j)$ and hence also $(i, j) \in C_{i^{\prime}}$.

The above argument means that the components $C_{i}$, with $i \in S$ form a partition of the graph $G$. Let us name $S_{1}=\left\{i \in S \mid C_{i}\right.$ ends at $\left.j(i) \in S\right\}$ and $S_{2}=\{i \in S \mid$ $C_{i}$ ends at $\left.j(i) \notin S\right\}$. Then,

$$
\begin{aligned}
& w^{*}(S)=w(N)-w(N \backslash S)=\sum_{(i, j) \in \mu} a_{i j}-\sum_{(i, j) \in \mu^{\prime}} a_{i j} \\
& =\sum_{i^{\prime} \in S_{1}}\left[\sum_{i \in C_{i^{\prime}}} a_{i \mu(i)}-\sum_{i \in C_{i^{\prime}} \backslash\left\{i^{\prime}\right\}} a_{i \mu^{\prime}(i)}\right]+\sum_{i^{\prime} \in S_{2}}\left[\sum_{i \in C_{i^{\prime}}} a_{i \mu(i)}-\sum_{i \in C_{i^{\prime}} \backslash\left\{i^{\prime}\right\}} a_{i \mu^{\prime}(i)}\right] \\
& \geq \sum_{i^{\prime} \in S_{1}} w^{*}\left(\left(i^{\prime}, j\left(i^{\prime}\right)\right)\right)+\sum_{i^{\prime} \in S_{2}} w^{*}\left(\left(i^{\prime}, 0\right)\right),
\end{aligned}
$$

where the inequality follows from (15) and (16), which completes the proof.

As a consequence of the above theorem, the set of dual coalitionally rational allocations of an assignment game can be defined only in terms of the dual constraints for mixed-pair and individual coalitions. Let us define for (extended) matrix $A=\left[a_{i j}\right]_{i, j \in M_{0}}$ a dual matrix $B=B(A)=\left[b_{i j}\right]_{i, j \in M_{0}}$ consisting of the dual coalitional values $b_{i j}=w_{A}^{*}((i, j))$ for all $i, j \in M_{0}$, including $b_{00}=0=w_{A}^{*}((0,0))$. 
With this notation, the set of dual coalitionally rational payoff vectors in $\left(N, w_{A}\right)$ is simply

$$
\mathcal{R}^{*}\left(N, w_{A}\right)=\left\{(u, v) \in \mathbb{R}^{M_{0}} \times \mathbb{R}^{M_{0}}: u_{i}+v_{j} \leq b_{i j} \text { for all } i, j \in M_{0}\right\} .
$$

Notice that $b_{i i}=a_{i i}$ for all $i \in M_{0}$, so to use this description of $\mathcal{R}^{*}\left(N, w_{A}\right)$ (or the dual description (13) of the core) only the $b_{i j}=w_{A}(N)-w_{A}(N \backslash\{i, j\})$ values for $i \neq j \in M_{0}$ need to be computed from the underlying matrix $A$.

Recall that the individual and mixed-pair upper bounds collected in matrix $B$ are attained in the core. Hence no entry in $B$ can be decreased without modifying the core of the game and $B$ is the minimum matrix among those representing the same core. Consequently, the dual matrix $B$ satisfies a dual form of the exactness property (12).

Proposition 6 Let $\left(N, w_{A}\right)$ be a square assignment game with an optimal matching on the main diagonal. Then, the dual matrix $B=B(A)$ satisfies

$$
b_{i j}+b_{k k} \leq b_{i k}+b_{k j} \quad \text { for all } i, j, k \in M_{0} .
$$

Proof. Let $(u, v) \in \mathcal{C}\left(N, w_{A}\right)$ be the core element that attains, for an arbitrarily chosen pair $i, j \in M_{0}$, the upper bound $b_{i j}$, that is, $u_{i}+v_{j}=b_{i j}$. Then, $b_{i j}+b_{k k}=$ $u_{i}+v_{j}+u_{k}+v_{k} \leq b_{i k}+b_{k j}$ holds for any $k \in M_{0}$.

\section{Computation of the essential dual coalitional values}

We now turn to the question of how to determine the dual matrix $B=B(A)$. The diagonal entries are obviously $b_{i i}=a_{i i}$ for all $i \in M_{0}$. To obtain the off-diagonal entries by their definition, we must compute each of the $(m+1) m$ optimum values $w_{A}(N \backslash\{i, j\}), i \neq j \in M_{0}$. Since solving an $m \times m$ assignment optimization problem takes, in general, $\mathcal{O}\left(\mathrm{m}^{3}\right)$ time, this straightforward approach would require $\mathcal{O}\left(\mathrm{m}^{5}\right)$ time. Alternatively, we could use the tightness of the dual upper bounds and obtain $b_{i j}$ as the optimum value of the linear programming problem $\max \left\{u_{i}+v_{j}:(u, v) \in\right.$ $\left.\mathcal{C}\left(N, w_{A}\right)\right\}$, but the computational complexity of this approach is not known to us. Next we present an $\mathcal{O}\left(\mathrm{m}^{4}\right)$ method that requires no optimization.

Suppose we already know the (extended) matrix $\bar{A}=\left[\bar{a}_{i j}\right]_{i, j \in M_{0}}$ of the exact core lower bounds, that is, for any $i, j \in M_{0}$ there exists a core vector $(u, v)$ such that $u_{i}+v_{j}=\bar{a}_{i j}$. Obviously, $\bar{a}_{i i}=a_{i i}$ for all $i \in M_{0}$. We claim that the matrix of exact core upper bounds $B(A)$ is given by

$$
b_{i j}=\bar{a}_{i i}+\bar{a}_{j j}-\bar{a}_{j i}, \quad \text { for all }(i, j) \in M_{0} \times M_{0} .
$$

Indeed, for all core vectors $(u, v)$ and all $i, j \in M_{0}$ we have $u_{i}+v_{j}=u_{i}+v_{i}+u_{j}+$ $v_{j}-u_{j}-v_{i} \leq \bar{a}_{i i}+\bar{a}_{j j}-\bar{a}_{j i}$. To see that this upper bound is attained, take a core vector $(u, v)$ for which $u_{j}+v_{i}=\bar{a}_{j i}$. Thus, $\bar{a}_{i i}+\bar{a}_{j j}-\bar{a}_{j i}$ is the exact upper bound for core payoffs $u_{i}+v_{j}$, implying our claim (18).

Notice that it only takes $\mathcal{O}\left(\mathrm{m}^{2}\right)$ elementary operations to obtain matrix $B(A)$ from matrix $\bar{A}$ via (18). Combined with the $\mathcal{O}\left(\mathrm{m}^{4}\right)$ algorithm we propose next to compute $\bar{A}$ from the initial matrix $A$, we obtain a method to compute $B(A)$ from 
$A$ that is faster than the aformentioned direct approaches. Moreover, our method requires only elementary operations and no optimization.

\section{Algorithm Cover}

Initially, let $A^{0}=A_{M_{0} \times M_{0}}$ be a square matrix with an optimal matching in the diagonal. Set $r=1$.

Iteration $r$ : compute matrix $A^{r}$ from matrix $A^{r-1}$ as follows:

$$
a_{i j}^{r}:=\max \left\{a_{i j}^{r-1}, \max \left\{a_{i k}^{r-1}+a_{k j}^{r-1}-a_{k k}^{r-1}: k \neq i, j \in M_{0}\right\}\right\} \quad \text { for all } i, j \in M_{0} .
$$

If $A^{r}=A^{r-1}$ then STOP, else set $r:=r+1$ and start a new iteration.

Output: matrix $\bar{A}=A^{\bar{r}}$ where $\bar{r}$ is the first $r \geq 1$ for which $A^{r}=A^{r-1}$.

In Proposition 7 below we show that the algorithm terminates after a finite number of iterations, but we need some preparation. Given a square matrix $A$ with an optimal matching in the diagonal, we say that entry $a_{i j}$ is (strictly) majorized by a loop of length $r$ if there is a sequence of $r$ distinct indices $k_{1}, \ldots, k_{r}$, all of them different from $i$ and $j$, such that $a_{i j}(<) \leq a_{i k_{1}}-a_{k_{1} k_{1}}+a_{k_{1} k_{2}}-a_{k_{2} k_{2}}+\ldots-a_{k_{r} k_{r}}+$ $a_{k_{r} j}$. We say that an $a_{i j}$-majorizing loop is stronger than another $a_{i j}$-majorizing loop (irrespective of their length), if its value (= the alternating sum of the loop entries) is higher than the value of the other loop. We remark that

1. by the optimality of the diagonal, none of its entries $a_{i i}$ can be strictly majorized by any loop, and that a loop which weakly majorizes $a_{i i}$ gives an alternative optimal assignment for $A$;

2. the exactness condition (12) means that no entry of the matrix is strictly majorized by a loop of length 1 ;

3. the exactness condition (12) implies that no entry of the matrix is strictly majorized by a loop of any length. Indeed, suppose the strict majorization $a_{i j}<a_{i k_{1}}-a_{k_{1} k_{1}}+a_{k_{1} k_{2}}-a_{k_{2} k_{2}}+\ldots-a_{k_{r} k_{r}}+a_{k_{r} j}$ by a loop of length $r \geq 2$. Under (12), the first three terms of the right hand side is $\leq a_{i k_{2}}$, revealing the strict majorization $a_{i j}<a_{i k_{2}}-a_{k_{2} k_{2}}+a_{k_{2} k_{3}}-\ldots-a_{k_{r} k_{r}}+a_{k_{r} j}$ by a loop of length $r-1$. Sequentially repeating the above argument reveals the strict majorization $a_{i j}<a_{i k_{r}}-a_{k_{r} k_{r}}+a_{k_{r} j}$ by a loop of length 1 , a contradiction to (12).

Now we are ready to show the correctness of the algorithm.

Proposition 7 In algorithm COVER

1. the number of iterations is $\bar{r} \leq m$;

2. the number of elementary operations is $\mathcal{O}\left(m^{4}\right)$.

Proof. Obviously, for any $r \geq 1, a_{i j}^{r} \geq a_{i j}^{r-1}$ for all $i, j \in M_{0}$, hence the name of the algorithm. By the above remark 1, the diagonal entries are never increased, $a_{i i}^{r}=a_{i i}^{r-1}$ for all $i \in M_{0}$.

Claim 1 follows from two observations. The first one is that if an entry $a_{i j}$ of the input matrix $A^{0}=A_{M_{0} \times M_{0}}$ is strictly majorized by a loop that contains an entry 
$a_{p q}$ then $a_{i j}$ can not be part of any loop that (strictly) majorizes $a_{p q}$. Indeed, let, for example, $a_{i j}<a_{i k_{1}}-a_{k_{1} k_{1}}+a_{k_{1} k_{2}}-a_{k_{2} k_{2}}+a_{k_{2}=p, k_{3}=q}-a_{k_{3} k_{3}}+a_{k_{3} k_{4}}-a_{k_{4} k_{4}}+a_{k_{4} j}$, and at the same time, $a_{p q} \leq a_{p s_{1}}-a_{s_{1} s_{1}}+a_{s_{1}=i, s_{2}=j}-a_{s_{2} s_{2}}+a_{s_{2} s_{3}}-a_{s_{3} s_{3}}+a_{s_{3} q}$. By adding these two inequalities and cancelling $a_{i j}+a_{p q}$ from both sides, we get $0<\left[a_{i k_{1}}-a_{k_{1} k_{1}}+a_{k_{1} k_{2}}-a_{k_{2}, k_{2}=p}\right]+\left[a_{p s_{1}}-a_{s_{1}, s_{1}=i}\right]+\left[-a_{q=k_{3}, k_{3}}+a_{k_{3} k_{4}}-a_{k_{4} k_{4}}+\right.$ $\left.a_{k_{4} j}\right]+\left[-a_{j=s_{2}, s_{2}}+a_{s_{2} s_{3}}-a_{s_{3} s_{3}}+a_{s_{3}, q}\right]$, where the first and third brackets contain the terms from the first inequality separated by $a_{p q}$, while the second and fourth brackets contain the terms from the second inequality separated by $a_{i j}$. However, the sum of the terms in the first and second brackets is $\leq 0$ by the optimality of the diagonal assignment and, similarly, the sum of the terms in the third and fourth brackets is also $\leq 0$ by the same reason. Thus, the sum of the terms in all four brackets is $\leq 0$. This contradiction proves the acyclicity of the (mixed strict and weak) loop-majorization relation between the matrix entries.

The second observation is that (19) eliminates strict majorizations by loops of length 1 in the current matrix $A^{r-1}$. Although it may create strict majorization by loops of length 1 of the same entry in the updated matrix $A^{r}$, but only if a longer and stronger majorizing loop existed in $A^{r-1}$. Indeed, it may even happen that an entry is not increased in an iteration, but it must be increased in the next or some subsequent iteration(s). Assume, for example, $a_{i j}^{r-1}=a_{i j}^{r}$, but $a_{i j}^{r}<$ $a_{i j}^{r+1}=a_{i k_{1}}^{r}-a_{k_{1} k_{1}}^{r}+a_{k_{1} j}^{r}$ because the entry $a_{i k_{1}}^{r-1}<a_{i k_{1}}^{r}=a_{i k_{2}}^{r-1}-a_{k_{2} k_{2}}^{r-1}+a_{k_{2} k_{1}}^{r-1}$ had to be increased in iteration $r$. Substituting $a_{i k_{1}}^{r}$ with this loop value in the above expression that defines $a_{i j}^{r+1}$, we get $a_{i j}^{r}<a_{i k_{2}}^{r-1}-a_{k_{2} k_{2}}^{r-1}+a_{k_{2} k_{1}}^{r-1}-a_{k_{1} k_{1}}^{r}+a_{k_{1} j}^{r}$. By replacing $a_{k_{1} k_{1}}^{r}=a_{k_{1} k_{1}}^{r-1}$ and $a_{k_{1} j}^{r}$ with its defining expression in (19), we get a strictly majorizing longer loop in $A^{r-1}$ that is stronger than any of the loops of length 1. Since (19) strictly reduces the length of the majorizing loops that exist in the current matrix $A^{r-1}$, and by definition their length is at most $m$, Claim 1 follows.

To see Claim 2, notice that updating an entry by (19) takes $\mathcal{O}(m)$ time, $\mathcal{O}\left(m^{2}\right)$ entries need to be updated, so each iteration takes $\mathcal{O}\left(\mathrm{m}^{3}\right)$ time. By Claim 1, the number of iterations is at most $m$, so algorithm Cover takes $\mathcal{O}\left(m^{4}\right)$ time.

Before we prove that algorithm COVER determines matrix $\bar{A}=\left[\bar{a}_{i j}\right]_{i, j \in M_{0}}$ of the exact core lower bounds, we illustrate it on the following example.

Example 3 Let the assignment game with row agents $I=\{1,2,3\}$ and columns agents $J=\left\{1^{\prime}, 2^{\prime}, 3^{\prime}\right\}$ be induced by the following extended matrix $A$. The value of the grand coalition is given by the optimal assignment in the diagonal.

$$
A=\left[\begin{array}{c||ccc}
\hline 0 & 0 & 0 & 0 \\
\hline 0 & 5 & 2 & 2 \\
0 & 6 & 5 & 1 \\
0 & 3 & 4 & 3
\end{array}\right] .
$$

When computing matrix $A^{1}$ from the initial $A^{0}=A$ in iteration $r=1$, we find that matrix $A$ violates the exactness property (12) for several combination of indices, so we increase, if necessary, the off-diagonal entries of $A^{0}$ as little as needed to satisfy property (12). For example, $a_{01}^{1}=1=\max \left\{a_{02}^{0}+a_{21}^{0}-a_{22}^{0}=0+6-5=\right.$ $\left.1, a_{03}^{0}+a_{31}^{0}-a_{33}^{0}=0+3-3=0\right\}$. Similarly, $a_{31}^{1}=5=\max \left\{a_{21}^{0}+a_{32}^{0}-a_{22}^{0}=\right.$ 
$\left.6+4-5=5, a_{30}^{0}+a_{01}^{0}-a_{00}^{0}=0\right\}$. We get

$$
A^{1}=\left[\begin{array}{c||ccc}
0 & 1 & 1 & 0 \\
\hline 0 & 5 & 3 & 2 \\
1 & 6 & 5 & 3 \\
0 & 5 & 4 & 3
\end{array}\right],
$$

set $r:=2$, and start a new iteration.

In iteration $r=2$ we check whether $A^{1}$ violates property (12) by computing matrix $A^{2}$ and checking whether there is an entry to be increased. There is only one, namely, $a_{01}^{2}=2=\max \left\{a_{02}^{1}+a_{21}^{1}-a_{22}^{1}=1+6-5=2, a_{03}^{1}+a_{31}^{1}-a_{33}^{1}=\right.$ $0+5-3=2\}$. We get

$$
A^{2}=\left[\begin{array}{c||ccc}
0 & 2 & 1 & 0 \\
\hline 0 & 5 & 3 & 2 \\
1 & 6 & 5 & 3 \\
0 & 5 & 4 & 3
\end{array}\right],
$$

set $r:=3$, and start a new iteration.

In iteration $r=3$ we find that $A^{3}=A^{2}$, so it satisfies the exactness property (12). The algorithm stops after $\bar{r}=3(\leq m=3)$ iterations.

The reader can easily check that in this example the entries of the output matrix $A^{3}=A^{2}$ are precisely the exact core lower bounds, so indeed $\bar{A}=A^{3}$ and the matrix

$$
B=\left[\begin{array}{c||ccc}
0 & 5 & 4 & 3 \\
\hline 3 & 5 & 4 & 3 \\
4 & 7 & 5 & 4 \\
3 & 6 & 5 & 3
\end{array}\right]
$$

obtained from $\bar{A}$ by (18) consists of the exact core upper bounds. Notice that at the margins we obtained the individual core upper bounds for all players. No optimization was needed to compute the dual game values.

Now we show that algorithm Cover determines the exact core lower bounds for coalitions in family $\mathcal{B}$ given in (10). It will be convenient to refer to the solution set of the system in (11) for any non-negative square matrix $A_{M_{0} \times M_{0}}$ with an optimal assignment on the main diagonal as the cover of matrix $A$, and denote it by $\mathcal{C}(A)$. Since we might judiciously think of $\mathcal{C}(A)$ as the core of a generalized assignment game (Owen, 1992), where the individual values are not necessarily all zero, but $a_{i 0} \geq 0$ for $i \in I$ and $a_{0 j} \geq 0$ for $j \in J$, using the same notation as for the core of the game should not be confusing. Naturally, $\mathcal{C}(A)=\mathcal{C}\left(N, w_{A}\right)$ in case $A_{M_{0} \times M_{0}}$ is the standard extension with zeros of the mixed-pair value matrix $A_{I \times J}$.

Proposition 8 In any iteration $1 \leq r \leq \bar{r}$ of algorithm COVER,

1. $\mathcal{C}\left(A^{r}\right)=\mathcal{C}\left(A^{r-1}\right) \neq \varnothing$;

2. $A^{r}$ is diagonal-optimal (i.e. its main diagonal forms an optimal assignment).

Proof. We simultaneously prove the claims by induction. Obviously, at the start of the first iteration $r=1$, we have $\mathcal{C}\left(A^{r-1}\right) \neq \varnothing$ and $A^{r-1}$ is diagonal-optimal. Now we assume that both statements hold at the start of arbitrary iteration $r \geq 1$. 
To see Claim 1, first recall that $a_{i j}^{r} \geq a_{i j}^{r-1}$ for all $i, j \in M_{0}$ by (19), and $a_{i i}^{r}=a_{i i}^{r-1}$ for all $i \in M_{0}$ by the diagonal-optimality of $A^{r-1}$. This immediately implies $\mathcal{C}\left(A^{r}\right) \subseteq \mathcal{C}\left(A^{r-1}\right)$. To show the reverse inclusion, take any $(u, v) \in \mathcal{C}\left(A^{r-1}\right)$ and $i, j \in M_{0}$. Then we have $u_{i}+v_{j}=u_{i}+v_{k}+u_{k}+v_{j}-\left(u_{k}+v_{k}\right) \geq a_{i k}^{r-1}+a_{k j}^{r-1}-a_{k k}^{r-1}$ for any $k \neq i, j \in M_{0}$, and also $u_{i}+v_{j} \geq a_{i j}^{r-1}$, hence $u_{i}+v_{j} \geq a_{i j}^{r}$ by (19). On the other hand, $u_{i}+v_{i}=a_{i i}^{r-1}=a_{i i}^{r}$ for all $i \in M_{0}$, thus $\mathcal{C}\left(A^{r}\right) \supseteq \mathcal{C}\left(A^{r-1}\right)$ also holds, implying $\mathcal{C}\left(A^{r}\right) \neq \varnothing$.

To see Claim 2, take any $(u, v) \in \mathcal{C}\left(A^{r}\right) \neq \varnothing$. Then $\sum_{k=0}^{m} a_{k k}^{r}=\sum_{k=0}^{m}\left(u_{k}+\right.$ $\left.v_{k}\right)=\sum_{(i, j) \in \mu}\left(u_{i}+v_{j}\right) \geq \sum_{(i, j) \in \mu} a_{i j}^{r}$ for any full matching $\mu \in \mathcal{M}\left(I \cup\{0\}, J \cup\left\{0^{\prime}\right\}\right)$, where 0 and $0^{\prime}$ denotes the fictitious row and column player, respectively.

Notice that the output matrix $\bar{A}=A^{\bar{r}}$ defines the unique generalized assignment game in sense of Owen (1992) which is exact and has the same core as the original assignment game induced by the input matrix $A$.

\section{Lemaral vectors and extreme core points}

With the above efficient and elementary way of computing the dual matrix $B(A)$ at hand, let us see how to use it to obtain the set of extreme core allocations of an assignment game.

Theorem 1 shows that, given an assignment game $\left(N, w_{A}\right)$, the lemaral payoff vectors are computed from expression (8) with $\mathcal{D}=\mathcal{B}$ defined in (10). In order to be consistent with our conventions in representing a single player as a mixed pair with the artificial player of the other type, we need to adjust the general definitions as follows. Let $I_{0}=I \cup\{0\}$ and $J_{0}=J \cup\left\{0^{\prime}\right\}$ denote the extended sets of row and column players, respectively. We will consider only those orders of the extended player set $N_{0}=I_{0} \cup J_{0}$ in which the first two positions are occupied by the artificial players 0 and $0^{\prime}$. Let $\Pi_{0}\left(N_{0}\right)$ denote their set. Notice that the orders in $\Pi_{0}\left(N_{0}\right)$ are in one-to-one correspondence with the orders in $\Pi(N)$, thus $\left|\Pi_{0}\left(N_{0}\right)\right|=(2 m)$ ! although $\left|N_{0}\right|=2 m+2$.

The lemarals are obtained from the dual matrix $B_{M_{0} \times M_{0}}$ in the following way. For each order $\sigma \in \Pi_{0}\left(N_{0}\right)$, the corresponding lemaral $\bar{r}^{\sigma, w_{A}} \in \mathbb{R}^{N_{0}}$ is iteratively defined by $\bar{r}_{\sigma_{1}}^{\sigma, w_{A}}=\bar{r}_{\sigma_{2}}^{\sigma, w_{A}}=0$ and, for all $k=3, \ldots, 2 m+2$ by

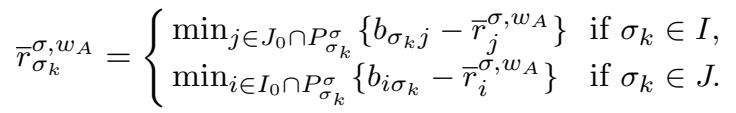

Recall that we require $P_{\sigma_{3}}^{\sigma}=\left\{0,0^{\prime}\right\}$. Notice that expression (20) is indeed the specialization of (8) with $\mathcal{D}=\mathcal{B}$ to assignment games, thus for each order $\sigma \in$ $\Pi_{0}\left(N_{0}\right)$, the corresponding payoff vector is indeed the lemaral $\bar{r}^{\sigma, w_{A}}$.

Since assignment games are ONTO-marginal (Hamers et al., 2002), a consequence of Proposition 4 is that assignment games are also ONTO-lemaral, that is, each extreme core allocation is a lemaral payoff vector. The next theorem shows that assignment games are also INTO-lemaral.

Theorem 2 Let $\left(N, w_{A}\right)$ be the assignment game induced by matrix $A_{M_{0} \times M_{0}}$. For each order $\sigma \in \Pi_{0}\left(N_{0}\right)$, the lemaral payoff vector $\bar{r}^{\sigma, w_{A}}$ is an extreme point of $\mathcal{C}\left(N, w_{A}\right)$. 
Proof. Since any lemaral which is in the core is an extreme point of the core, we only need to show that all lemarals are in the core. Since for each $\sigma \in \Pi_{0}\left(N_{0}\right)$ we have $\bar{r}^{\sigma, w_{A}} \in \mathcal{R}^{*}\left(N, w_{A}\right)$ by definition, we only need to check efficiency to prove $\bar{r}^{\sigma, w_{A}} \in \mathcal{C}\left(N, w_{A}\right)$. To make notation easier, let us write $\bar{r}^{\sigma, w_{A}}=(u, v)$.

To this end, let $\mu=\left\{(k, k): k \in M_{0}\right\}$ be an optimal matching in $A$. By definition, $\bar{r}_{k}^{\sigma, w_{A}}=0$ for both $k=0$ and $k=0^{\prime}$, so $u_{0}+v_{0}=b_{00}$. Now take a pair $(k, k)$ for some $k \in M$. Let us assume without loss of generality that $\sigma^{-1}(\mu(k))>$ $\sigma^{-1}(k)$, i.e. row player $k$ precedes column player $k^{\prime}$. We want to prove that $b_{k k}-$ $u_{k} \leq b_{i k}-u_{i}$ for all $i \in P_{\mu(k)}^{\sigma} \cap I_{0}$, which means that $b_{k k}-u_{k}$ is the minimum in (20) for $v_{k}=\bar{r}_{\mu(k)}^{\sigma, w_{A}}$, implying $u_{k}+v_{k}=b_{k k}$.

To this end, take any $i \in P_{\mu(k)}^{\sigma} \cap I_{0}$, and assume that $u_{k}=b_{k j}-v_{j}$ for some $j \in P_{k}^{\sigma} \cap J_{0}$. We consider two cases. If $\sigma^{-1}(i)>\sigma^{-1}(j)$ then $j \in P_{i}^{\sigma} \cap J_{0}$ and

$$
b_{k k}-u_{k}=b_{k k}-\left(b_{k j}-v_{j}\right) \leq b_{i k}-\left(b_{i j}-v_{j}\right) \leq b_{i k}-u_{i},
$$

where the first inequality follows from $b_{i j}+b_{k k} \leq b_{i k}+b_{k j}$, which holds by Proposition 6 , and the second inequality from $u_{i}+v_{j} \leq b_{i j}$.

Otherwise, that is, if $\sigma^{-1}(i)<\sigma^{-1}(j)$, then $v_{j} \leq b_{i j}-u_{i}$ and hence

$$
b_{k k}-u_{k}=b_{k k}-b_{k j}+v_{j} \leq b_{k k}-b_{k j}+b_{i j}-u_{i} \leq b_{i k}-u_{i},
$$

where the last inequality follows again from Proposition 6.

The above theorem also enables us to simplify the computation of the lemarals. Since any lemaral is now proved to be in the core of the assignment game, once we determine the payoff to a player in (20), the payoff to his optimally assigned partner is also determined by their efficiency equation $u_{k}+v_{k}=b_{k k}=a_{k k}$. The next example illustrates this simplified way of computing the lemarals.

Example 4 Consider the assignment game and its dual matrix $B$ in Example 3. We compute the lemaral $\bar{r}^{\sigma}$ related to the order $\sigma=\left(3^{\prime}, 3,2^{\prime}, 2,1,1^{\prime}\right)$ (for brevity, we omit the artificial players in the first two positions, since always $\bar{r}_{0}^{\sigma}=0$ and $\left.\bar{r}_{0^{\prime}}^{\sigma}=0\right)$ :

$$
\begin{array}{ll}
\bar{r}_{3^{\prime}}^{\sigma}=b_{03}=3 & \text { and } \bar{r}_{3}^{\sigma}=3-3=0, \\
\bar{r}_{2^{\prime}}^{\sigma}=\min \left\{b_{02}, b_{32}-\bar{r}_{3}^{\sigma}\right\}=4 & \text { and } \bar{r}_{2}^{\sigma}=5-4=1, \\
\bar{r}_{1}^{\sigma}=\min \left\{b_{10}, b_{12}-\bar{r}_{2^{\prime}}^{\sigma}, b_{13}-\bar{r}_{3^{\prime}}^{\sigma}\right\}=0 & \text { and } \bar{r}_{1^{\prime}}^{\sigma}=5-0=5 .
\end{array}
$$

Hence, $\bar{r}^{\sigma}=(0,1,0 ; 5,4,3)$. It is easily checked to be the extreme core allocation that gives all sellers their core minimum payoffs, see the output matrix $A^{2}$ in Example 3.

Having seen that for assignment games the set of extreme core allocations coincides with the set of lemarals, and thus by Proposition ?? with the set of lemacols, we get that Algorithm COVER together with (18) and (20) give a simple and quite efficient way to compute the extreme core allocations, or the lemacols, directly from the extended matrix $A^{0}$.

In order to further emphasize the relevance of Theorem 2, we give a game whose core shows very strong similarities with the core of an assignment game, but has an extreme core element that is neither a lemacol, nor a lemicol vector. 
Example 5 Consider the 6-player game $(N, v)$ with

$$
v(S)=\left\{\begin{array}{rll}
12 & \text { if } S=N \\
4 & \text { if } S \in\{\{1,4\},\{2,5\},\{3,6\}\} \\
3 & \text { if } S=\{2,3\} \\
4 & \text { if } S \in\{\{1,2,3\},\{1,2,6\}\} \\
0 & \text { otherwise }
\end{array}\right.
$$

In any core allocation $x_{1}+x_{4}=4, x_{2}+x_{5}=4, x_{3}+x_{6}=4$, so we can picture the core in the $\left(x_{1}, x_{2}, x_{3}\right)$-space, see Figure 1 . Moreover, $x_{i} \geq 0, i=1, \ldots, 6$,

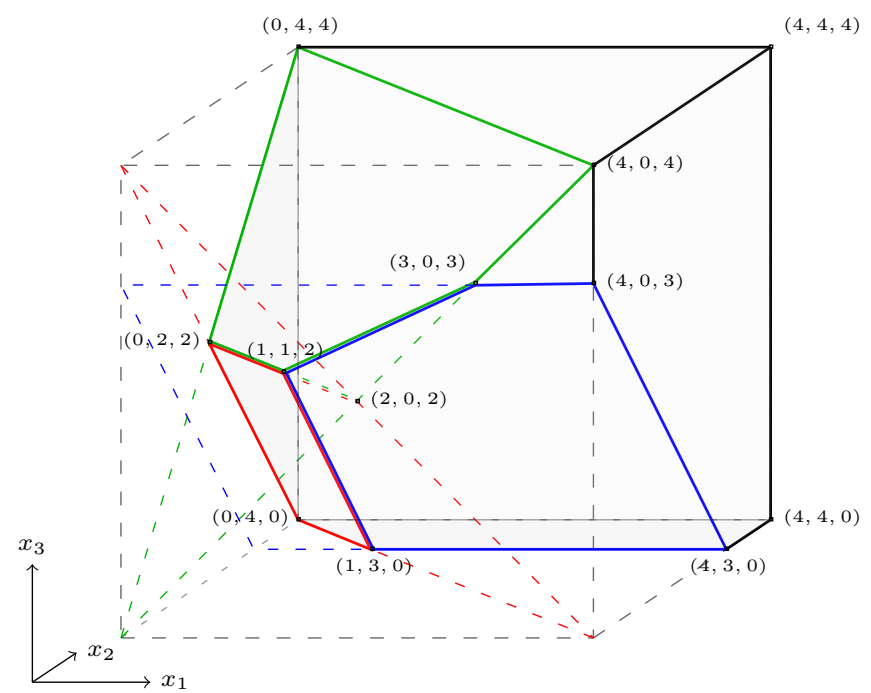

Fig. 1 The (projection of the) core in Example 5

thus the (projection to that space of the) core is included in the box $0 \leq x_{i} \leq 4$, $i=1,2,3$.

The inequality $x_{1}+x_{2}+x_{3} \geq 4$ cuts off corner $(0,0,0)$, the inequality $x_{1}+$ $x_{2}+x_{6} \geq 4$ cuts off corner $(0,0,4)$. These two planes intersect in the line segment joining the points $(0,2,2)$ (the midpoint of the left-side square), and $(2,0,2)$ (the midpoint of the front side-square). The inequality $x_{2}+x_{3} \geq 3$ cuts off this latter point and the corner $(4,0,0)$. The above-mentioned three planes intersect in the point $(1,1,2)$.

The point $(1,1,2)$ is an extreme point of the core (it makes 6 linearly independent core inequalities binding). All other core extreme points (shown) are on the sides of the box (at least one of their coordinates is 0 or 4 , the core-minimum or the core-maximum payoff, respectively, for each variable). Therefore, the core extreme point $(1,1,2)$ is neither a lemacol, nor a lemicol. It cannot even be obtained by any mixed sequence of maximization / minimization within a given order (e.g. max for the first player, min for the second and for the third, again max for the fourth, etc.). 
Notice, however, that because of the dichotomous relation between the pairs of payoffs $x_{i}$ and $x_{3+i}=4-x_{i}$ for $i=1,2,3$ inside the core, all but the $(1,1,2)$ core extreme points are actually lemacols (and also lemicols).

\section{Concluding remarks for the average lexicographical values}

An immediate consequence of the above results regarding the computation of the lemarals is that we have obtained an easy way to compute the average of all lemacol payoff vectors, that is the AL-value.

As the reader will realize after computing several lemarals for our Example 4 , many of them coincide. The reason is that once the payoff to an agent in a given order $\sigma$ is fixed, the position that his optimally assigned partner occupies among his followers does not matter. Thus we can restrict ourselves to those orders where optimally assigned partners are consecutive. Let $\mu$ be an optimal assignment, $\mu \in \mathcal{M}_{A}^{*}(I, J)$, and define

$\tilde{\Pi}(N)=\left\{\sigma \in \Pi(N):\right.$ for all $i \in I$, if $\sigma^{-1}(i)=r$ then $\left.\sigma^{-1}(\mu(i)) \in\{r-1, r+1\}\right\}$.

Notice that for $(m+m)$-player assignment games, i.e. when $|N|=|I \cup J|=2 m$, the cardinality of $\tilde{\Pi}(N)$ is $m ! \cdot 2^{m}$, much less than $(2 m)$ !, the cardinality of $\Pi(N)$. In the above $(3+3)$-player example, we would only need to consider 48 orders instead of 720 . For $(4+4)$-player assignment games, the respective numbers are 384 versus 40320 .

In light of the above remark, the expression of the AL-value for assignment game $\left(N, w_{A}\right)$ can be rewritten as follows:

$$
A L\left(w_{A}\right)=\frac{1}{m ! \cdot 2^{m}} \sum_{\sigma \in \tilde{\Pi}(N)} \bar{r}^{\sigma, w_{A}} .
$$

The inequalities $m ! \cdot 2^{m}<m ! \cdot m^{m}<(2 m)$ ! and the fact that it is more efficient to compute a lemaral than a marginal payoff vector show that for assignment games the AL-value is computationally far more tractable than the Shapley value. Moreover, we believe that the number of orders for which the lemaral is computed in $(21)$ can be further decreased. Since the number of extreme core points in an $(m+m)$-player assignment game is at most $\left(\begin{array}{c}2 m \\ m\end{array}\right)$ (Balinski and Gale, 1987), and $\left(\begin{array}{c}2 m \\ m\end{array}\right)<(m+1) ! \leq m ! \cdot 2^{m}$, several lemacols related to orders even in $\tilde{\Pi}(N)$ must coincide in the same extreme core point. Further studies are needed to see how these groups of orders (and their multiplicity) could be determined.

Moreover, the AL-value and the reverse AL-value coincide in the case of assignment games. This is because the lemacol/lemicol for an order $\sigma=\left(\sigma_{1}, \sigma_{2}, \ldots, \sigma_{n}\right)$ of the players is the same as the lemicol/lemacol for the related order

$$
\tilde{\sigma}=\left(\mu\left(\sigma_{1}\right), \mu\left(\sigma_{2}\right), \ldots, \mu\left(\sigma_{n}\right)\right)
$$

Notice that $\tilde{\sigma}$ keeps the order between pairs as in $\sigma$ but reverses the order of the players within each pair: if for example $\sigma=\left(1,2^{\prime}, 3,3^{\prime}, 1^{\prime}, 2\right)$, then $\tilde{\sigma}=\left(1^{\prime}, 2,3^{\prime}, 3,1,2^{\prime}\right)$. 
The reason is that, over any subset of the core, when $i \in M_{0}$ attains her maximum payoff, the partner $\mu(i)$ attains his minimum one. Formally, since $\underline{\lambda}_{\sigma_{1}}^{\sigma, w}=$ $\min \left\{x_{\sigma_{i}}: x \in C(N, w)\right\}$, we have

$$
\underline{\lambda}_{\mu\left(\sigma_{1}\right)}^{\sigma, w}=\max \left\{x_{\sigma_{1}}: x \in C(N, w)\right\}=\bar{\lambda}_{\mu\left(\sigma_{1}\right)}^{\tilde{\sigma}, w} .
$$

In the core, once fixed the payoff to $\sigma_{1}$, the payoff to the partner $\mu\left(\sigma_{1}\right)$ is fixed simultaneously. Hence, we have that, if $\sigma_{2} \neq \mu\left(\sigma_{1}\right)$, then

$$
\begin{aligned}
\underline{\lambda}_{\sigma_{2}}^{\sigma, w} & =\min \left\{x_{\sigma_{2}}: x \in C(N, w), x_{\sigma_{1}}=\underline{\lambda}_{\sigma_{1}}^{\sigma, w}\right\} \\
& =\min \left\{x_{\sigma_{2}}: x \in C(N, w), x_{\sigma_{1}}=\underline{\lambda}_{\sigma_{1}}^{\sigma, w}, x_{\mu\left(\sigma_{1}\right)}=\underline{\lambda}_{\mu\left(\sigma_{1}\right)}^{\sigma, w}\right\} .
\end{aligned}
$$

As a consequence,

$$
\begin{aligned}
\underline{\lambda}_{\mu\left(\sigma_{2}\right)}^{\sigma, w} & =\max \left\{x_{\mu\left(\sigma_{2}\right)}: x \in C(N, w), x_{\sigma_{1}}=\underline{\lambda}_{\sigma_{1}}^{\sigma, w}, x_{\mu\left(\sigma_{1}\right)}=\underline{\lambda}_{\mu\left(\sigma_{1}\right)}^{\sigma, w}\right\} \\
& =\max \left\{x_{\mu\left(\sigma_{2}\right)}: x \in C(N, w), x_{\mu\left(\sigma_{1}\right)}=\bar{\lambda}_{\mu\left(\sigma_{1}\right)}^{\tilde{\sigma}, w}=\bar{\lambda}_{\mu\left(\sigma_{2}\right)}^{\tilde{\sigma}, w} .\right.
\end{aligned}
$$

By iteration of the above argument we obtain $\underline{\lambda}_{k}^{\sigma, w}=\bar{\lambda}_{k}^{\tilde{\sigma}, w}$ for all $k \in N$. As a consequence, the AL-value coincides with the reverse AL-value.

\section{References}

1. Balinski ML, Gale D (1987) On the core of the assignment game. In: Functional Analysis, Optimization, and Mathematical Economics, ed. Leifman LJ, Oxford University Press, New York, 274-289.

2. Biswas A, Parthasarathy T, Potters JAM, Voorneveld M (1999) Large cores and exactness. Games and Economic Behavior, 20:1-12.

3. Demange G (1982) Strategyproofness in the assignment market game. Mimeo, Laboratoire d'Econométrie de l'École Politechnique, Paris.

4. Estévez-Fernández A (2012) New characterizations for largeness of the core. Games and Economic Behavior 76: 160-180.

5. Gellekom JRG van, Potters JAM, Reijnierse JH (1999) Prosperity properties of TU-games. International Journal of Game Theory, 28:211-227.

6. Ichiishi, T (1981) Supermodularity: applications to convex games and to the greedy algorithm for LP. Journal of Economic Theory, 25:283-286.

7. Izquierdo JM, Núñez M, Rafels C (2007) A simple procedure to obtain the extreme core allocations of an assignment market. International Journal of Game Theory, 36:17-26.

8. Leonard HB (1983) Elicitation of honest preferences for the assignment of individuals to positions. Journal of Political Economy, 91: 461-479.

9. Hamers H, Klijn F, Solymosi T, Tijs S, Villar JP (2002) Assignment games satisfy the CoMa-property. Games and Economic Behavior, 38:231-239.

10. Kuipers J (1993) On the core of information graph games. International Journal of Game Theory, 21:339-350.

11. Kongo T, Funaki Y, Branzei R, Tijs S (2010) Non-cooperative and axiomatic characterizations of the average lexicographic value. International Game Theory Review, 12(4):417-435. (doi: 10.1142/S0219198910002751)

12. Martínez-de-Albéniz J, Núñez M, Rafels C (2011) Assignment markets with the same core. Games and Economic Behavior, 73(2): 553-563.

13. Núñez M, Rafels C (2002) Buyer-seller exactness in the assignment game. International Journal of Game Theory, 31:423-436.

14. Owen, G. (1992) The assignment game: The reduced game. Annales D'Economie et de Statistique, 25/26:71-79.

15. Shapley LS (1971) Cores of convex games. International Journal of Game Theory, 1:11-26.

16. Shapley LS, Shubik M (1972) The assignment game I: The core. International Journal of Game Theory, 1:111-130. 
17. Solymosi T, Raghavan T (2001) Assignment games with stable core. International Journal of Game Theory, 30:177-185.

18. Tijs S, Borm P, Lohmann E, Quant M (2011) An average lexicographic value for cooperative games. European Journal of Operational Research, 213:210-220. 